\title{
Angiosarcoma after adjuvant radiotherapy in high-risk squamous cell carcinoma of the vulva: a case report
}

\author{
Concetta Laliscia' ${ }^{1}$, Angiolo Gadducci ${ }^{2}$, Luca Emanuele Pollina ${ }^{3}$, Fabiola Paiar ${ }^{1}$ \\ ${ }^{1}$ Department of New Technologies and Translational Research, Division of Radiation Oncology, University of Pisa, Pisa, Italy \\ ${ }^{2}$ Department of Clinical and Experimental Medicine, Division of Gynecology and Obstetrics, University of Pisa, Pisa, Italy \\ ${ }^{3}$ Division of Pathology, University Hospital of Pisa, University of Pisa, Pisa, Italy
}

\begin{abstract}
Squamous cell carcinoma of the vulva represents 3-5\% of gynecological cancers. The incidence is higher in postmenopausal patients; the mean age of women with vulvar cancer is between 64 and 70 years.

Radiotherapy plays an increasing role in the treatment of high-risk squamous cell carcinoma of the vulva; associated with surgery it significantly improves prognosis but is also associated with serious late side-effects, such as secondary malignancies. We describe a case of a 75-year-old woman who underwent deep total vulvectomy with inguinal-femoral lymphadenectomy for high-risk, keratinizing variant HPV-negative, squamous cell carcinoma of the vulva, followed by adjuvant concomitant chemo-radiotherapy, at the University Hospital of Pisa in February 2013. Five years later she developed a very large angiosarcoma in the right abdominal wall, at the edge of the previous radiotherapy field, and underwent radical surgery. After four months, she developed bone metastasis of angiosarcoma, also treated with surgery.

This experience shows that the use of new technologies allows the delivery of high doses of radiotherapy, significantly correlated with a better prognosis, but also associated with fortunately rare morbidity, such as radiation-induced angiosarcoma. Due to the presence of long, mostly post-menopausal survivors among irradiated patients, screening for second malignancies must be developed for selected high-risk survivor groups.
\end{abstract}

Key words: radiotherapy, vulvar carcinoma, angiosarcoma, surgery.

\section{Introduction}

Squamous cell carcinoma of the vulva represents $3-5 \%$ of gynecological cancers. The incidence is higher in old and postmenopausal patients; the mean age of women with vulvar cancer is between 64 and 70 years. A long retrospective Iranian study reported that vulvar cancer was significantly more common in multiparous $(p<0.001)$ and menopausal patients $(p<0.001)[1,2]$.

Primary and secondary angiosarcoma is a rare malignant endothelial-cell tumor of vascular or lymphatic origin; the incidence is 12,000 new cases in the US per year, with approximately 5,000 deaths, but recently it is increasing with the use of radiotherapy [3-5]. Indeed, the US National Cancer Institute's Surveillance, Epidemiology and End Results (SEER) program reported that second malignancies have doubled in the last three decades (9\% in $1975-1979$ to $19 \%$ in $2005-2009$ ) [6].

The number of long-term cancer survivors has significantly increased; the reasons include better lifestyle, genetic susceptibility, results of tailored systemic che- motherapy and increased use of advanced technologies in radiation therapy, as also described in the literature on breast cancer [7]. But many studies show that anti-cancer treatments can potentially induce new primary or second malignancies [5].

A Memorial Sloan Kettering Cancer Center (MSKCC) study reported that a time range of six months is sufficient to diagnose radiation-induced sarcoma (RIS), in contrast with the generally accepted median time range of eleven years (range 3-36) [8].

\section{Case report}

A 75-year-old woman underwent deep total vulvectomy with inguino-femoral lymphadenectomy for high-risk keratinizing variant HPV-negative, squamous cell carcinoma of the vulva (pT1bN2aM0) followed by adjuvant concomitant chemo-radiotherapy, at the University Hospital of Pisa in February 2013. Treatment planning was established after exhaustive discussion 
between gynecological oncologists and radiotherapists within a multidisciplinary team.

External beam radiotherapy (EBRT) using 6-MeV photons was delivered with volumetric modulated arc therapy (VMAT). The target clinical volume included the vulvar tumor bed, bilateral inguinal-femoral, external and internal iliac nodes (Fig. 1). The total EBRT dose was 50.4Gy in 6 weeks, daily fractions of $1.8 \mathrm{~Gy}$, with weekly concomitant cisplatin (CDDP) $40 \mathrm{mg} / \mathrm{m}^{2}$ for five cycles. Acute and late toxicity was graded according to the Common Terminology Criteria for Adverse Events (CTCAE) version 5.0 [9]. Adverse events were grade 2 dermatitis, grade 1 diarrhea and neutropenia. Subsequent follow-up showed no evidence of disease, until November 2015 when a computed tomography (CT) scan and a clinical examination showed local recurrence $(15 \mathrm{~mm})$ and so the woman underwent local surgical excision.

Subsequent follow-up revealed no evidence of recurrence until March 2018, when she presented with a hard, dark red large tumor mass $(87 \mathrm{~mm} \times 53 \mathrm{~mm} \times 30 \mathrm{~mm}$ ) with multiple red-violaceus satellite nodules extending from skin to subcutaneous tissue, in the right lower abdominal wall, just cranial to the pubis. No other lymph node or distant metastasis was observed (Fig. 2). Then the patient underwent wide surgical resection with a negative margin.

Histopathological assessment showed cutaneous and subcutaneous multicenter infiltrating angiosarcoma and microscopically demonstrated a mesenchymal proliferation consisting of moderately atypical cells and frequent endothelial mitoses (CD34+, CD31+, HHV8-, Ckpan-) (Fig. 3).

Four months later a CT scan showed bone metastasis at the left femur and the woman underwent surgery followed by physiotherapy. Histopatological assessment showed infiltrating bone tissue angiosarcoma (CD31+, CD34+, podoplanin and actin 1a4-).

The patient has been followed up until April 2019 and is alive without evidence of new disease.

\section{Discussion}

RISs are rare malignancies with a poor prognosis: five-year survival varies between $17 \%$ and $58 \%$ [8]. Angiosarcomas are $56.8 \%$ of the sarcomas occurring in the fields of prior radiotherapy, as well as in a large SEER study of breast cancer patients with second malignancies [10].

Post-radiation sarcomas arise expecially in the area contained by the $5 \%$ isodose line, or at the edge of the radiotherapy field. The lower radiation dose at the edge of the radiation field may be not be able to kill all cells, but can lead to cell damage and genetic mutations. Exposure to low dose radiation can cause double DNA breaks (DSBs), which could lead to gene mutations and malignant transformation of the irradiated cell. Radiation doses < 0.2 Gy fail to activate the G2/M cell cycle check point so DNA damage is not repaired and carcinogenesis is generated [11].

However, RIS is also observed at high doses of radiation up to $45 \mathrm{~Gy}$, probably caused by accelerated repopulation of pre-malignant cells during and after radiotherapy, potentially in contrast with the effects of

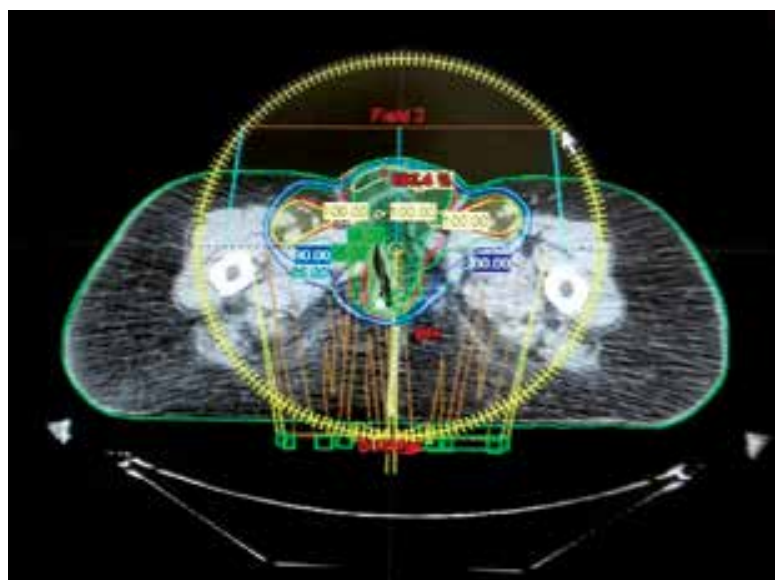

Fig. 1. Volumetric arc therapy (VMAT) in high-risk squamous cell carcinoma of the vulva

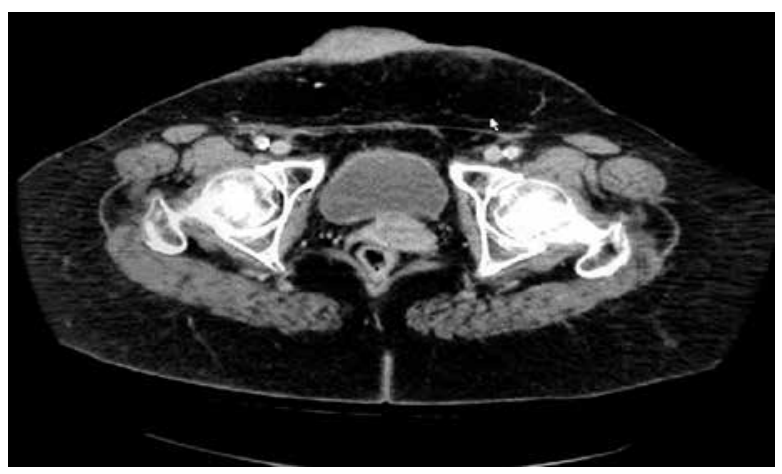

Fig. 2. Preoperative computed tomography scan of the abdomen

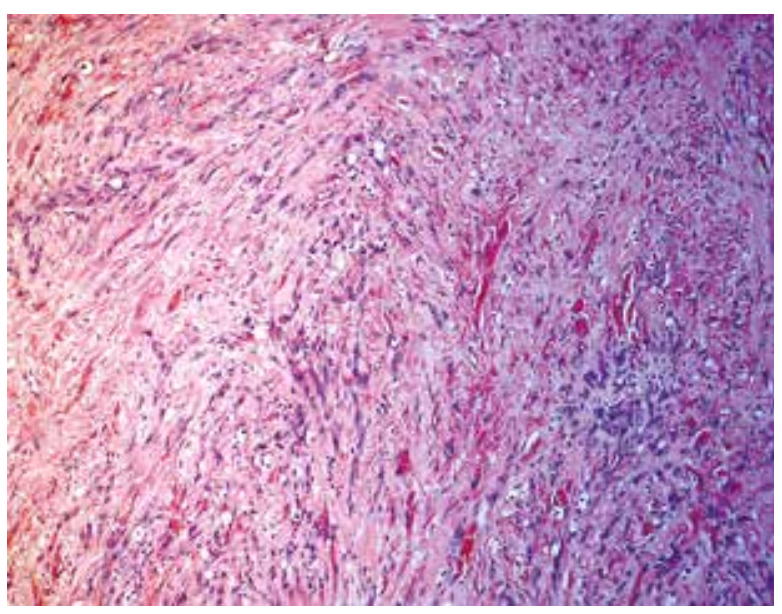

Fig. 3. Angiosarcoma 
cell killing at higher doses. The pathogenesis must be due to lethal chromosomal damage in connective tissue cells that occasionally generate clinical sarcoma in or at the edge of the radiation field [5].

Use of older radiation techniques has been found to increase the risk of RIS as well as recently new radiation techniques such as intensity modulated-radiation therapy (IMRT) or image-guided radiation therapy (IGRT). Using IMRT, small volumes are exposed to a high dose, and large volumes of normal tissue are exposed to a lower dose, resulting in a higher total dose delivered [12].

Moreover, use of IGRT during set-up verification contributes to about $5-20 \%$ of the total dose to normal tissues, outside treatment fields, potentially increasing the long-term risk of RIS, especially in younger patients [13].

An Italian review reported a lower second malignancy rate in patients treated with hadrontherapy compared to patients treated with conventional radiotherapy: $5.2 \%$ vs. $7.5 \%$ in a study of Chung et al. The probable reason is that the dose deposited by protons ends sharply near the end of their range, while the dose deposition by photons is less conformal $[14,15]$.

Radical surgery with negative histological margins (RO) is the only curative therapy for localized disease, while radiotherapy and chemotherapy have a minor role in the treatment [16].

\section{Conclusions}

Radiation-induced angiosarcoma is considered a rare but serious late side-effect associated with radiation therapy for primary malignancies, including gynecological cancers. However, most of the cases described in the literature are associated with breast cancer, less with gynecological malignancies, particularly with vulvar cancer [17]. The use of new technologies allows the delivery of high doses of radiation therapy, significantly correlated with a better prognosis, but also potentially associated with rare significant morbidity such as secondary malignancies.

Due to the presence of long, mostly post-menopausal survivors among irradiated patients, screening for second malignancies (especially breast cancer) has been developed for selected high-risk survivor groups.

\section{References}

1. Butt JL, Botha MH. Vulvar cancer is not a disease of the elderly: Treatment and outcome at a tertiary referral centre in South Africa. S Afr Med 2017; 107: 1000-1004

2. Mousavi A, Yousefnezhad A, Modarres-Gilani M, et al. Vulvar cancer in Iran: Retrospective study over 20 years (1998-2018). Family Med Prim Care 2019; 8: 1465-1469.

3. Wibmer C, Leithner A, Zielonke N, et al. Increasing incidence rates of soft tissue sarcomas? A population-based epidemiologic study and literature review. Ann Oncol 2010; 21: 1106-1111.

4. Siegel RL, Miller KD, Jemal A. Cancer statistics. CA Cancer J Clin 2015; 65: 5-29.

5. Dracham CB, Shankar A, Madan R. Radiation induced secondary malignancies: a review article. Radiat Oncol J 2018; 36: 85-94.

6. Morton LM, Onel K, Curtis RE, et al. The rising incidence of second cancers: patterns of occurrence and identification of risk factors for children and adults. Am Soc Clin Oncol Educ Book 2014: e57-67.

7. Neuhaus SJ, Pinnock N, Giblin V, et al. Treatment and outcome of radiation-induced soft-tissue sarcomas at a specialist institution. Eur J Surg Oncol 2009; 35: 654-659.

8. Gladdy RA, Qin LX, Moraco N, et al. Do radiation-associated soft tissue sarcomas have the same prognosis as sporadic soft tissue sarcomas? J Clin Oncol. 2010; 28: 2064-2069.

9. National Institutes of Heatlh. Common Terminology Criteria for Adverse Events (CTCAE), 2017.

10. Huang J, Mackillop WJ. Increased risk of soft tissue sarcoma after radiotherapy in women with breast carcinoma. Cancer 2001; 92: 172-180.

11. Mullenders L, Atkinson $\mathrm{M}$, Paretzke $\mathrm{H}$, et al. Assessing cancer risks of low-dose radiation. Nat Rev Cancer 2009; 9: 596-604.

12. Hall EJ. Intensity-modulated radiation therapy, protons, and the risk of second cancers. Int J Radiat Oncol Biol Phys 2006; 65: 1-7.

13. Newhauser WD, Durante M. Assessing the risk of second malignancies after modern radiotherapy. Nat Rev Cancer 2011; 11: 438-448.

14. Chung CS, Yock TI, Nelson K, et al. Incidence of second malignancies among patients treated with proton versus photon radiation. Int J Radiat Oncol Biol Phys 2013; 87: 46-52.

15. Facoetti A, Barcellini A, Valvo F, et al. The Role of Particle Therapy in the Risk of Radio-induced Second Tumors: A Review of the Literature. Anticancer Res 2019; 39: 4613-4617.

16. Libshitz HI. Radiation changes in bone. Semin Roentgenol 1994; 29: 15-37.

17. Shin YB, Choi WJ, Kim C. Radiation-induced cutaneous angiosarcoma of the abdomen in a patient with cervical cancer: A case report. J Obstet Gynaecol Res 2019; 45: 1941-1943.

\section{Disclosure}

The authors report no conflict of interest. 